\title{
DEPRESSÃO E FOBIA SOCIAL: UM CASO CLÍNICO DE AVALIAÇÃO E INTERVENÇÃO COGNITIVO- COMPORTAMENTAL
}

DEPRESSION AND SOCIAL PHOBIA: A CLINICAL CASE STUDY OF COGNITIVE-BEHAVIORAL THERAPY EVALUATION AND INTERVENTION

DEPRESIÓN Y FOBIA SOCIAL: UN CASO CLÍNICO DE EVALUACIÓN Y INTERVENCIÓN COGNITIVO-CONDUCTUAL

Luis Manuel Oliveira Carneiro*

\begin{abstract}
RESUMO
Este artigo pretende dar a conhecer os resultados duma intervenção cognitivo-comportamental num caso clínico de um jovem com problemas de violência doméstica. Apresenta sintomas depressivos que o prejudicam a nível pessoal, acadêmico e social. Para além disso, manifesta medo e ansiedade acerca de diversas situações de âmbito social em que é exposto à possível avaliação por parte dos outros e receio de que possa vir a comportarse de modo embaraçador. A avaliação psicológica permitiu diagnosticar uma perturbação depressiva e fobia social, tendo sido acionada a intervenção cognitivo-comportamental com base no modelo cognitivo para a depressão, de Beck, e no modelo cognitivo para a ansiedade social, de Clark e Wells. Os resultados da intervenção foram positivos, indicando a importância da terapia cognitivo-comportamental nesses tipos de perturbação.
\end{abstract}

Palavras-chave: Depressão. Fobia social. Avaliação psicológica. Terapia cognitivo-comportamental. Estudo de caso.

\section{ABSTRACT}

The aim of this paper is to present the results of a cognitive-behavioral therapy clinical case report of a young man with domestic violence issues. The subject shows depressive symptoms that affect him on a personal, academic and social level. Furthermore, he shows fear and anxiety about several social situations in which he may fall under the scrutiny from other people, and worries about the likelihood of behaving in an embarrassing manner. With the psychological assessment, the subject was diagnosed with depression and social phobia, and cognitive-behavioral therapy based on Beck's Cognitive Model of Depression and Clark \& Wells's Cognitive Model for Social Anxiety were started off. The results of the intervention

\footnotetext{
Texto recebido em 27 de maio de 2017 e aprovado para publicação em 12 de agosto de 2019.

Mestre em Psicologia, Área de Especialização em Psicologia da Saúde, pela Escola de Psicologia - Universidade do Minho, Portugal; pós-graduado em Psicoterapias Cognitivo-Comportamentais, pelo Instituto Português de Psicologia e Outras Ciências (Inspsic), Porto, Portugal. Lapsis - Centro de Apoio Psicoterapêutico e Psicopedagógico, Ltda. Endereço: Rua General Bernardo do Canto, 11. 9560-106 Lagoa, Açores, São Miguel, Portugal. E-mail: luis.m.o.carneiro@gmail.com.
} 
were positive, indicating the importance of cognitive-behavioral therapy in this kind of disorders.

Keywords: Depression. Social phobia. Psychological assessment. Cognitivebehavioral therapy. Clinical case study.

\section{RESUMEN}

Este artículo pretende dar a conocer los resultados de una intervención cognitivo-conductual en un caso clínico de un joven con problemas de violencia doméstica. Él presenta síntomas depresivos que lo perjudican a nivel personal, académico y social. Más allá de eso, presenta miedo y ansiedad sobre muchas situaciones sociales en las que es expuesto a la posible evaluación por parte de los demás y miedo de comportarse de manera embarazosa. La evaluación psicológica ha permitido diagnosticar un disturbio depresivo y fobia social, y fue accionada la intervención cognitivoconductual basada en el Modelo Cognitivo de la depresión de Beck y en el Modelo Cognitivo para la ansiedad social de Clark y Wells. Los resultados de la intervención fueron positivos, indicando la importancia de la terapia cognitivo-conductual en este tipo de disturbios.

Palabras clave: Depresión. Fobia social. Evaluación psicológica. Terapia cognitivo-conductual. Estudio de caso.

\section{INTRODUÇÃO}

\subsection{Depressão}

depressão, isto é, sentir-se em baixo e triste, é uma resposta normal a uma
perda, desilusão ou fracasso. Contudo existem depressões clinicamente
significativas que podem comprometer ao ponto de produzirem efeitos negativos ao nível familiar, social, acadêmico e profissional, podendo ser, inclusive, mortais (Klein, \& Wender, 2005; Knaus, 2006). Existem várias classificações das perturbaçôes depressivas segundo o Manual Diagnóstico e Estatístico das Perturbações Mentais (DSM-5) (American Psychiatric Association, 2014), sendo uma delas a perturbação depressiva persistente (distimia). A distimia é um estado de depressão crônica (Klein, \& Wender, 2005), sendo que os dois critérios de diagnóstico centrais, segundo o DSM-5, são:

a) humor deprimido, na maior parte do dia, na maioria dos dias, pelo período de, pelo menos, dois anos; 
b) presença de duas ou mais das seguintes características:

1) diminuição do apetite ou alimentação excessiva;

2) insônia ou hipersônia;

3) baixa energia ou fadiga;

4) baixa autoestima;

5) dificuldades de concentração ou em tomar decisões; e

6) sentimentos de desesperança.

Podem ocorrer episódios depressivos major (pelo menos duas semanas e com uma intensidade e frequência elevadas) antes ou durante a distimia (American Psychiatric Association, 2014).

\subsection{Fobia social}

A maior parte das pessoas já experimentou um certo nível de ansiedade, preocupação ou medo, sendo características normais que contribuem para a sobrevivência do organismo (Clark, \& Beck, 2010; Pinto-Gouveia, 2000). Todavia, quando é produzido um efeito negativo significativo no funcionamento a nível pessoal, social, acadêmico, profissional ou noutras áreas importantes, é provável estar presente a fobia social. Para o diagnóstico, de acordo com o DSM5, destacam-se os principais critérios:

a) medo ou ansiedade intensos sobre uma ou mais situaçóes sociais em que o indivíduo está exposto à possível avaliação pelas pessoas (ex.: ser observado a comer, fazer uma apresentação);

b) a pessoa tem medo de se comportar de modo a demonstrar sintomas de ansiedade que serão avaliados de forma negativa (ex.: ser humilhante);

c) as situações de âmbito social provocam, quase sempre, medo ou ansiedade;

d) o indivíduo evita ou enfrenta as situações sociais com medo ou ansiedade intensos;

e) o medo ou ansiedade é desproporcional à verdadeira ameaça conferida pela situação social e o contexto sociocultural; 
f) o medo, ansiedade ou evitamento é persistente, durando normalmente mais de 6 meses (American Psychiatric Association, 2014).

\subsection{Terapia cognitivo-comportamental na depressão e na fobia social}

O caso clínico que neste trabalho é apresentado é um exemplo de como a perturbação depressiva e a fobia social coexistem, originando consequências negativas em vários níveis. Portanto, e no sentido de solucionar as problemáticas em causa, foi acionada a terapia cognitivo-comportamental. A eficácia da terapia cognitivo-comportamental na depressão tem sido tão significativa como a farmacoterapia ou outras modalidades de intervenção (Powell, Abreu, Oliveira, \& Sudak, 2008). A intervenção cognitivo-comportamental na fobia social tem sido também a abordagem preferencial e a que produz melhores benefícios, quando comparada com a farmacoterapia isolada (Clark, \& Beck, 2010).

Em suma, neste trabalho, será apresentado um caso clínico em que se inclui o diagnóstico efetuado, a formulação de caso cognitivo-comportamental (fatores predisponentes, precipitantes e de manutenção; conceitualização), a avaliação por exames complementares, a intervenção cognitivo-comportamental e as conclusões.

\section{CASO CLÍNICO}

\subsection{Dados biográficos}

Cliente H.; 16 anos; solteiro; $1^{\circ}$ ano de escolaridade; vive com os pais e irmão.

\subsection{Dificuldades atuais e história do problema}

H. apresenta, há cerca de dois anos e alguns meses, sintomatologia depressiva. A nível emocional, mostra tristeza frequente, sentimentos de solidão, irritabilidade e ansiedade (emocional), sendo que, em termos motivacionais, apresenta falta de iniciativa para realizar atividades. A nível comportamental, mostra isolamento social e pouco envolvimento em atividades agradáveis, apresentando sintomas somáticos como falta de apetite, tensão, fadiga muscular e perturbações do sono (ex.: ao adormecer). A nível cognitivo, evidencia desvalorização pessoal excessiva, dificuldades de concentração e falta de interesse pela vida/ideação suicida. Segundo o jovem, esses sintomas surgiram em resposta a um ambiente familiar 
disfuncional caracterizado pela ocorrência de discussóes frequentes. $\mathrm{O}$ motivo principal das discussões é, de acordo com H., o fato de este não desejar seguir a profissão do pai, lavrador, e, portanto, culpa-se frequentemente pelas agressões físicas do pai para com a mãe (ex.: "Se eu não existisse, não havia discussões"). Para além disso, há cerca de um mês, começaram a ocorrer discussóes entre o pai, a mãe e o irmão devido à descoberta de que este último consumia estupefacientes.

H. refere ainda outras dificuldades e problemas, nomeadamente medo de situações sociais (ex.: cafés, centros comerciais, hospitais, sala de aula). Nessas situaçóes, a nível emocional, sente-se extremamente ansioso e envergonhado e, a nível físico, cora, sente o coração acelerado, treme com as mãos e com o corpo e, por isso, tem evitado a maioria das situações sociais por achar que pode ser avaliado negativamente. Quando as enfrenta, tenta adotar comportamentos com o objetivo de minimizar a sua ansiedade e, ou, ajudar a "disfarçar" seu nervosismo (ex.: não olhar nos olhos das pessoas; segurar objetos com muita força). A nível cognitivo, $\mathrm{H}$. tem receio de que o possam avaliar negativamente ("vão pensar mal de mim") e lida mal com episódios em que pode estar sujeito à observação de outros (“já sabia que ia tropeçar”). Relativamente ao início dos sintomas, $\mathrm{H}$. referiu que, no $7^{\circ}$ ano, uma professora criticou-o numa aula por ter respondido incorretamente a uma questão colocada, tendo os colegas manifestado risos. A partir daí, deixou de participar nas aulas por medo de ser criticado e passou a evitar outras situações de âmbito social.

\subsection{História do desenvolvimento}

\subsubsection{Vida escolar, profissional e social}

Segundo a mãe, H. adaptou-se bem à escola, tinha facilidade em fazer amigos, brincava com todas as crianças e não mostrava isolamento social. Sempre foi bom aluno, nunca reprovou de ano, embora tivesse mais dificuldades em FísicoQuímica e Matemática, e tinha aulas de apoio de Português e de Matemática. A disciplina preferida era o Inglês. Quanto aos pais, a mãe era a única que manifestava disponibilidade e interesse para ajudar o filho nalgum assunto da escola, sendo que o pai apenas exigia que transitasse todos os anos ("é a sua obrigação"). A nível profissional, tem o desejo de ser técnico de informática. Em termos sociais, tem atualmente dois melhores amigos. As principais atividades de tempo livre são jogar futebol, estar no computador, jogar paintball e andar de bicicleta. Quanto a relações amorosas, já teve várias, mas duraram sempre pouco tempo. 


\subsubsection{Antecedentes familiares/ambiente familiar}

Segundo H., a mãe é uma pessoa carinhosa, preocupada e disponível, ajudando-o sempre que necessário. É considerada "superprotetora", que sempre alertou e se preocupou com os perigos, apresentando sintomas de depressão e de ansiedade social. Quanto ao pai, H. descreve-o como uma pessoa extremamente autoritária, que privilegia a ordem e o cumprimento rígido de regras. Dá primazia à exigência e ao controle das coisas, em detrimento do lazer e do bem-estar, e não expressa sentimentos. $\mathrm{H}$. refere que o pai nunca o elogia e que sempre o critica em vários assuntos, atendendo sempre aos aspetos negativos e punindo fisicamente. H. disse ainda que o pai valoriza muito "aquilo que os outros pensam" e a imagem que pode passar para os vizinhos, amigos e conhecidos da família.

Quanto ao casamento dos pais, $\mathrm{H}$ refere que nunca viu muito afeto na relação conjugal, notando a existência de pouca comunicação. Assistiu a algumas discussões, mas estas pioraram devido ao problema de dependências do irmão. A nível profissional, o pai é lavrador e a mãe é doméstica.

\subsection{Hipóteses diagnósticas/formulação}

\subsubsection{Diagnóstico}

Os dois diagnósticos atribuídos a $\mathrm{H}$ foram 300.4 (F34.1) Perturbação depressiva persistente (distimia), com sintomas ansiosos, início precoce, com episódios depressivos major intermitentes, com episódio atual, moderada e 300.23 (F40.10) Perturbação de ansiedade social (fobia social). Os diagnósticos foram feitos com base nos critérios indicados pelo DSM-5 (American Psychiatric Association, 2014).

\subsubsection{Fatores predisponentes, precipitantes e de manutenção}

Para uma melhor compreensão da predisposição, do início e da persistência das perturbações apresentadas por H., é essencial explorar os fatores predisponentes, precipitantes e de manutenção. Os fatores predisponentes da depressão incluem a falta de afeto do pai, seu estilo parental crítico e punitivo, seu desinteresse/ negligência e a restrição emocional. De fato, eventos de estresse, como negligência parental, aumentam o risco para a depressão (Nihalani, Simionescu, \& Dunlop, 2009). Essa relação entre o pai e H. permitiu a formação de crenças de defeito (ex.: 
"ninguém gosta de mim"), que aumentam a vulnerabilidade para a perturbação depressiva (Martin, \& Young, 2010; Sanderson, \& McGinn, 2001).

Salienta-se que esses fatores predisponentes assumem também um papel de manutenção dos sintomas depressivos atuais. $\mathrm{O}$ fato de a mãe também apresentar sintomas depressivos predispõe o desenvolvimento de depressão no filho (Klein, \& Wender, 2005). Nos fatores precipitantes da depressão, salientam-se as discussões familiares devido à discórdia de $\mathrm{H}$. em ser lavrador e a descoberta do consumo de substâncias por parte do irmão. Os fatores de manutenção incluem o ambiente familiar conflituoso e as interpretações negativas de $\mathrm{H}$. acerca de si e da situação familiar. De fato, existe uma maior probabilidade de desenvolver depressão para quem avalia de forma negativa certos eventos de vida significativos (Sanderson \& McGinn, 2001).

No que se refere aos fatores predisponentes da ansiedade social, é de referir o medo de avaliação negativa em situações sociais, característico da mãe, e o fato de esta ter sempre superprotegido o filho, que poderão ter tido uma influência significativa quanto à predisposição cognitiva do filho para a ansiedade social, incluindo a formação de esquemas de fracasso e de vulnerabilidade ao mal e à doença (Clark, \& Beck, 2010; Martin, \& Young, 2010). Para além disso, o fato de o pai de H. sempre se preocupar com o estatuto social da família pode ter conduzido $\mathrm{H}$. a formar esquemas negativos relacionados com a influência dos outros (ex.: "tenho sempre que parecer bem em situações sociais"), que constituem fatores predisponentes e de manutenção para a fobia social (Martin, \& Young, 2010). Salienta-se ainda que as crenças de defeito pessoal de H., formadas devido à relação com o pai, podem ter contribuído, de forma decisiva, para a formação e a manutenção de esquemas negativos de fracasso (ex.: "sou um desastrado"), uma vez que a literatura refere que experiências repetidas de humilhação ou negligência parecem contribuir para o desenvolvimento de vulnerabilidade cognitiva para a ansiedade (Clark, \& Beck, 2010; Martin, \& Young, 2010). Note-se que a baixa autoestima e autoconfiança, caraterísticas de H., também o predispóem para respostas autoprotetoras, como o evitamento (Clark, \& Beck, 2010). Relativamente aos fatores precipitantes da ansiedade social, o comportamento de evitamento social iniciou-se com o episódio de repreensão por parte de uma professora, no $7^{\circ}$ ano escolar. Consequentemente, e por medo da possível troça dos colegas, deixou de participar nas aulas e passou a ter receio de frequentar espaços sociais com muita gente, por medo de vir a comportar-se de maneira embaraçadora. Os fatores de manutenção da ansiedade social incluem a interpretação errada e distorcida das situações de interação social, o evitamento de situações sociais (ex.: a não participação nas aulas, evitar 
locais com muita gente) e a utilização de comportamentos de segurança (ex.: evitar o contacto visual) (Clark, \& Beck, 2010; Pinto-Gouveia, 2000).

\subsubsection{Conceitualização cognitivo-comportamental}

De acordo com o modelo cognitivo para a depressão de Beck (Beck, Rush, Shaw, \& Emery, 1979), a experiência precoce (ex.: pai crítico) conduz as pessoas à criação de crenças nucleares/esquemas disfuncionais sobre si mesmas ou sobre o mundo. Esses esquemas determinam a forma como o indivíduo interpreta a realidade, sendo sólidos e resistentes à mudança (ex.: "ninguém gosta de mim"). A essas crenças nucleares encontram-se associados pressupostos/regras que se traduzem em crenças condicionais (ex.: "se eu não existisse, não havia discussões"). Os esquemas podem ser ativados por situações semelhantes (ex.: críticas do pai) às experiências precoces que levaram à formação do esquema, sendo que essa ativação conduz ao aparecimento de pensamentos automáticos negativos (PAN), por exemplo: "eles discutem por culpa minha". Como tentativa de evitar a ativação dos esquemas, a pessoa pode usar estratégias compensatórias que permitem reduzir o sofrimento emocional temporariamente, já que, ao longo do tempo, podem contribuir para agravar as crenças (ex.: fazer as vontades ao pai). O significado atribuído aos PAN, de acordo com os esquemas, conduz a determinadas emoçôes (ex.: tristeza) e comportamentos (ex.: isolamento social). Os PAN resultam de erros de processamento cognitivo, pelos quais as interpretações das experiências são distorcidas (ex.: inferência arbitrária - acreditar que ninguém gosta dele e que não tem valor; abstração seletiva - percepcionar apenas situações negativas, esquecendo aspetos positivos; personalização assumir a culpa pelas discussões e violência entre os pais) (Knapp, \& Beck, 2008; Kunzler, \& Araujo, 2013; Mascella, 2013; Sanderson, \& McGinn, 2001; Wilkinson, Moore, \& Moore, 2003).

Segundo o modelo cognitivo de Clark e Wells (1995), quando um indivíduo entra numa situação social, é ativado um programa de ansiedade, que inclui três componentes que mantêm ou aumentam a ansiedade social. $\mathrm{O}$ primeiro componente abrange sintomas somáticos (ex.: taquicardia) e cognitivos (ex.: preocupação com a avaliação negativa dos outros) da ansiedade, que são ativados pela percepção de perigo, podendo estes ser encarados como nova fonte de perigo, gerando um ciclo vicioso. No segundo componente, o fóbico social focaliza sua atenção em si próprio (ex.: foco nos sintomas corporais e na possível avaliação dos outros). O terceiro componente refere-se aos comportamentos de segurança, que servem para diminuir a vivência de ameaça e o risco de avaliação negativa (ex.: evitar contato visual). O produto final do programa traduz-se na experiência 
de ansiedade, que leva a uma interação social ineficaz, que confirma os esquemas de ineficácia social, formando um ciclo de manutenção da fobia social (Clark, $\&$ Beck, 2010; Pinto-Gouveia, 2000). Ainda dentro desse modelo, o conceito de ansiedade antecipatória refere-se aos indivíduos que tentam reduzir seu receio de avaliação negativa, antecipando detalhadamente o que poderá acontecer na situação, originando níveis elevados de ansiedade que levam ao aumento da probabilidade de evitamento e de um desempenho social ineficaz e, portanto, à manutenção da fobia social (Clark, \& Beck, 2010; Pinto-Gouveia, 2000). Após a interação social, ocorre a autópsia, que inclui uma revisão pormenorizada do evento por parte do indivíduo, que avalia, de forma muito negativa, seu desempenho e recorda os fracassos prévios, fortalecendo seu autoesquema de incompetência/fracasso social (Clark, \& Beck, 2010; Pinto-Gouveia, 2000).

\subsection{Exames complementares de diagnóstico}

A avaliação psicológica de $\mathrm{H}$. foi complementada pela aplicação dos seguintes instrumentos psicométricos:

a) SCL-90-R (Derogatis, 1994);

b) STAI Forma Y1 e Y2 (Spielberger, 1983);

c) Inventário da Depressão de Beck (BDI) (McIntyre, \& McIntyre, 1995);

d) Escala de Ansiedade em Situações de Interação Social (SIAS) (Pinto-Gouveia, \& Salvador, 2001);

e) Escala de Fobia Social (SPS) (Pinto-Gouveia, \& Salvador, 2001);

f) Sheehan Disability Scale (SDS) (Pinto-Gouveia, Cunha, \& Salvador, 2000).

No geral, os resultados obtidos confirmaram muitas das queixas já descritas por $\mathrm{H}$. na entrevista clínica, por exemplo, sintomas depressivos (ex.: humor depressivo, desvalorização pessoal), agitação, tensão, sintomas físicos relacionados com a ansiedade social (ex.: taquicardia), medo de pessoas/locais, comportamentos de evitamento social e pensamentos negativos. 


\subsection{Intervenção na perturbação depressiva}

Tendo em conta o impacto emocional significativo e as consequências originadas pela depressão, a intervenção psicoterapêutica iniciou-se ao nível dessa perturbação. Portanto a terapia cognitiva para a depressão de Beck (Beck et al., 1979; Mascella, 2013) teve como objetivo principal procurar interromper o ciclo vicioso da depressão, treinando $\mathrm{H}$. a questionar seus pensamentos negativos automáticos e a combater as crenças nas quais se baseia (DeRubeis, Webb, Tang, \& Beck, 2010; Wilkinson et al., 2003). A intervenção foi feita semanalmente, durante 20 sessões, e quinzenalmente, durante 8 sessóes. $O$ período de followup incluiu 4 sessões: 2 dadas mensalmente, uma após três meses e uma após 6 meses.

Em primeiro lugar, foi descrito o modelo cognitivo de Beck (Beck et al., 1979). De forma a permitir uma melhor compreensão, foi construído o modelo do cliente (figura 1). Numa discussão pormenorizada com H., este reconheceu as relações entre suas crenças, os pensamentos, os sentimentos e os comportamentos disfuncionais resultantes (Knapp, \& Beck, 2008; Sanderson, \& McGinn, 2001; Wilkinson et al., 2003). 
Figura 1 - Modelo cognitivo de depressão do cliente H.

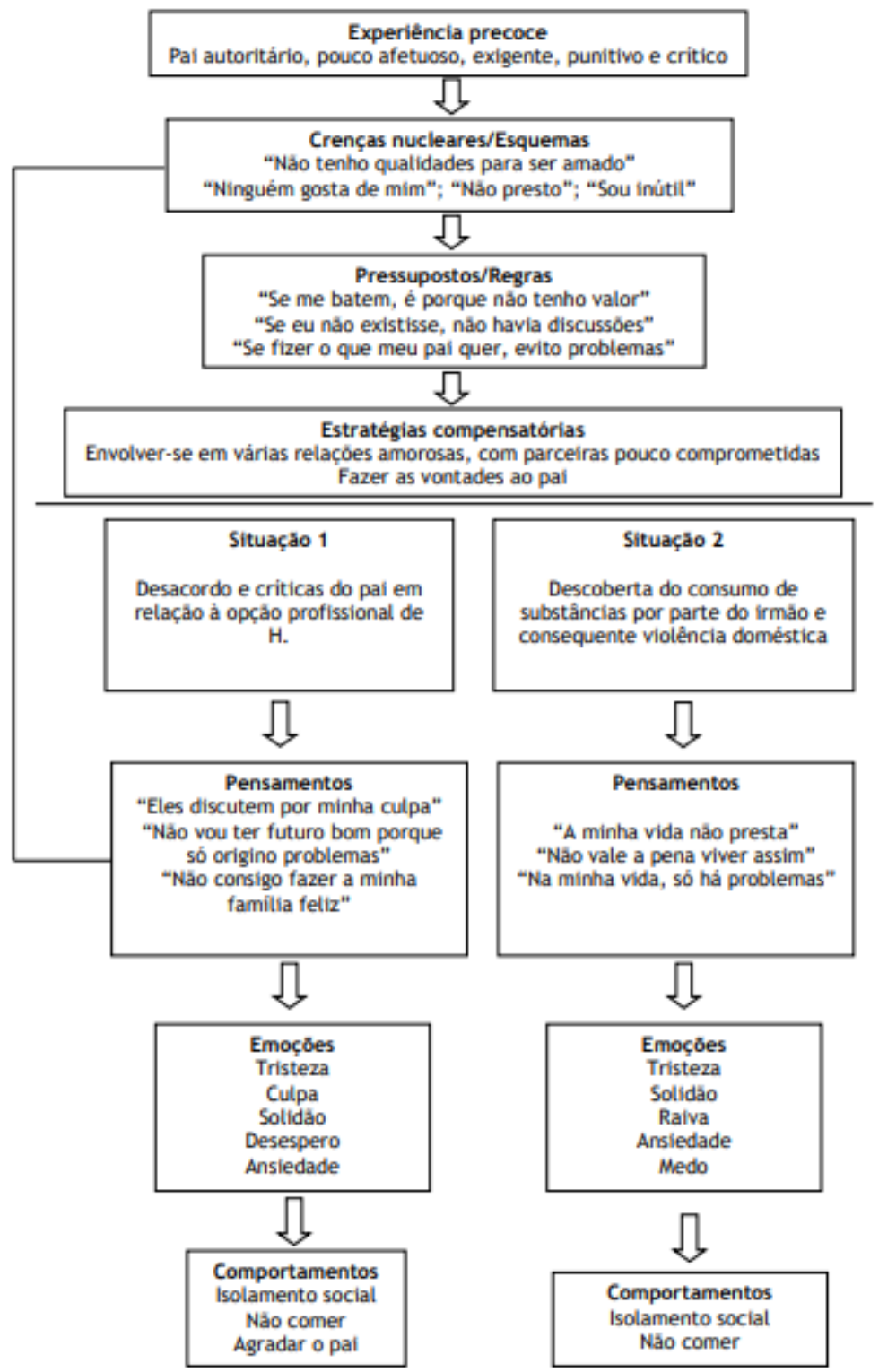


Em segundo lugar, iniciou-se a ativação comportamental no sentido de combater a falta de motivação nas atividades, a fadiga e o desânimo. Para tal, encorajou-se $\mathrm{H}$. a utilizar um mapa semanal de atividades para o controle e monitorização do nível e tipo de atividades que realiza. Semanalmente, foram programadas atividades que $\mathrm{H}$. deixou de realizar (ex.: sair com amigos) e solicitou-se o registo de seus progressos por meio de um mapa diário, que permite verificar e recordar os êxitos alcançados, em detrimento dos fracassos (DeRubeis et al., 2010; Sanderson, \& McGinn, 2001; Wilkinson et al., 2003).

Em terceiro lugar, procurou-se ensinar estratégias para a resolução da perda do apetite e consequente má alimentação, manifestadas por H. Posteriormente, auxiliou-se o cliente na questão das insônias, tendo mantido um registo diário do padrão de sono e aplicado estratégias para uma melhor higiene do sono (Ogden, 2010; Wilkinson et al., 2003).

Em quarto lugar, foi introduzida a técnica do relaxamento muscular progressivo, e sua prática foi encorajada, no sentido de melhorar a qualidade de sono e de reduzir os níveis de ansiedade/estresse e de tensão (Sanderson, \& McGinn, 2001; Sarafino, \& Smith, 2011).

Em quinto lugar, procedeu-se ao início do uso de estratégias cognitivas, tendo $\mathrm{H}$. sido incentivado a trazer à luz seus PAN, por meio de perguntas (questionamento socrático - QS) e das mudanças de humor durante as sessões. De modo a $\mathrm{H}$. tornar-se mais consciente da forma como os PAN influenciam seu humor, foi solicitado, como trabalho de casa, um registo das situações problemáticas, dos PAN e do grau de convicção nestes, das emoções, do grau de emocionalidade e dos comportamentos associados (DeRubeis et al., 2010; Sanderson, \& McGinn, 2001; Wilkinson et al., 2003).

Em sexto lugar, procedeu-se à análise da evidência dos PAN, por meio de questôes como: "qual é a evidência a favor do pensamento?", "qual é a evidência contra o pensamento?", "o que é que de pior poderia acontecer?", "quais os efeitos de pensar dessa maneira?", "quais as vantagens e desvantagens de pensar assim?", entre outras (DeRubeis et al., 2010; Sanderson, \& McGinn, 2001; Wilkinson et al., 2003).

Em sétimo lugar, passou-se, pela técnica da descoberta guiada (baseada no QS), à identificação de erros cognitivos, já mencionados inicialmente. H. foi também encorajado a fazer, como trabalho de casa, a análise de evidência para os PAN e a identificar os erros cognitivos (DeRubeis et al., 2010; Sanderson \& McGinn, 2001; Wilkinson et al., 2003). 
Em oitavo lugar, foi solicitado a $\mathrm{H}$. que gerasse explicações alternativas para os PAN apresentados, de modo a diminuir a rigidez do pensamento depressivo (DeRubeis et al., 2010; Gonçalves, 2004; Sanderson \& McGinn, 2001).

Em nono lugar, $\mathrm{H}$. foi instruído no treino da prática de respostas racionais às situações problemáticas. $\mathrm{O}$ trabalho de casa consistiu no registo das respostas positivas a seus PAN bem como do resultado. Nesse contexto, também foi utilizada a técnica do ponto/contraponto, que envolve a troca de papéis entre o psicólogo e H. em relação à defesa dos pensamentos negativos e à sua contraargumentação (DeRubeis et al., 2010; Gonçalves, 2004; Sanderson, \& McGinn, 2001; Wilkinson et al., 2003).

Em décimo lugar, e visando a testar a veracidade dos seus PAN, H. foi encorajado a envolver-se em experiências comportamentais, recorrendo-se a um registo, e também foram feitas perguntas por meio da técnica da descoberta guiada (Sanderson, \& McGinn, 2001).

Em décimo primeiro lugar, foi introduzido o treino de solução de problemas, tendo como objetivos definir bem o problema, pensar em várias soluções alternativas, avaliá-las em termos de prós e de contras, escolher a melhor e implementá-la, de forma a lidar, por exemplo, com as críticas do pai e com a ocorrência de discussōes familiares (Gonçalves, 2004; Nezu, Nezu, \& D'Zurilla, 2013; Sanderson, \& McGinn, 2001; Wilkinson et al., 2003).

Em décimo segundo e último lugar, procedeu-se à identificação e modificação dos esquemas disfuncionais que estão na base do surgimento dos PAN. Para tal, recorreu-se à terapia focada nos esquemas de Young (Young, 1990), uma vez que foi evidente a existência de um esquema maladaptivo precoce (EMP) de defeito. Nesse âmbito, foi importante a realização de técnicas experienciais com o objetivo de H. perceber o papel das experiências precoces na formação e fortalecimento de seus EMP, por meio da recordação de experiências passadas e atuais (ex.: críticas e relação com o pai), da expressão da raiva por diálogos imaginários (ex.: com o pai), da reparentização e da expressão emocional e de direitos por meio de cartas. Nos esquemas de H. (ex.: "ninguém gosta de mim"), foram utilizadas também técnicas cognitivas e comportamentais, como a análise de evidência, a identificação e classificação dos erros cognitivos, o treino de respostas racionais, a avaliação das vantagens e desvantagens das respostas de coping (ex.: desvantagens do isolamento social), o uso de experiências comportamentais, o emprego de flashcards com as qualidades de H. e a escolha de parceiras mais comprometidas, para evitar que a estratégia compensatória antiga continuasse a contribuir para a manutenção do EMP (DeRubeis et al., 2010; Gonçalves, 2004; Martin, \& Young, 2010; Sanderson, \& McGinn, 2001; Wilkinson et al., 2003). No fim 
da intervenção, H. foi capaz de produzir afirmações mais construtivas sobre seus esquemas (ex.: "consigo ver que há pessoas que gostam de mim e me dão valor").

\subsection{Intervenção na fobia social}

A intervenção psicoterapêutica ao nível da ansiedade social (Clark, \& Wells, 1995) teve a finalidade de colocar um termo ao ciclo da fobia social, visando-se à modificação dos pensamentos e das crenças negativas, à eliminação de comportamentos de segurança, do evitamento, do recurso à ansiedade antecipatória e à autópsia, e à alteração da percepção de perigo relacionada com as interaçôes e situações sociais. A intervenção envolveu 15 sessões semanais e 10 quinzenais, sendo que o período de follow-up englobou 4 sessões, 2 dadas mensalmente, uma após três meses e uma após 6 meses.

Numa primeira instância, foram explicados os conceitos teóricos de ansiedade e de medo, reforçando sua importância para a sobrevivência do organismo (Clark, \& Beck, 2010). Seguidamente, foi explicado o modelo cognitivo de Clark e Wells (1995), tendo sido construído um modelo simples de ansiedade social de H. (figura 2), de modo a facilitar sua compreensão e a verificar a existência de diversas interaçôes e de ciclos viciosos na fobia social (Clark, \& Beck, 2010; Pinto-Gouveia, 2000). 
Figura 2 - Modelo cognitivo de ansiedade social do cliente $\mathbf{H}$.

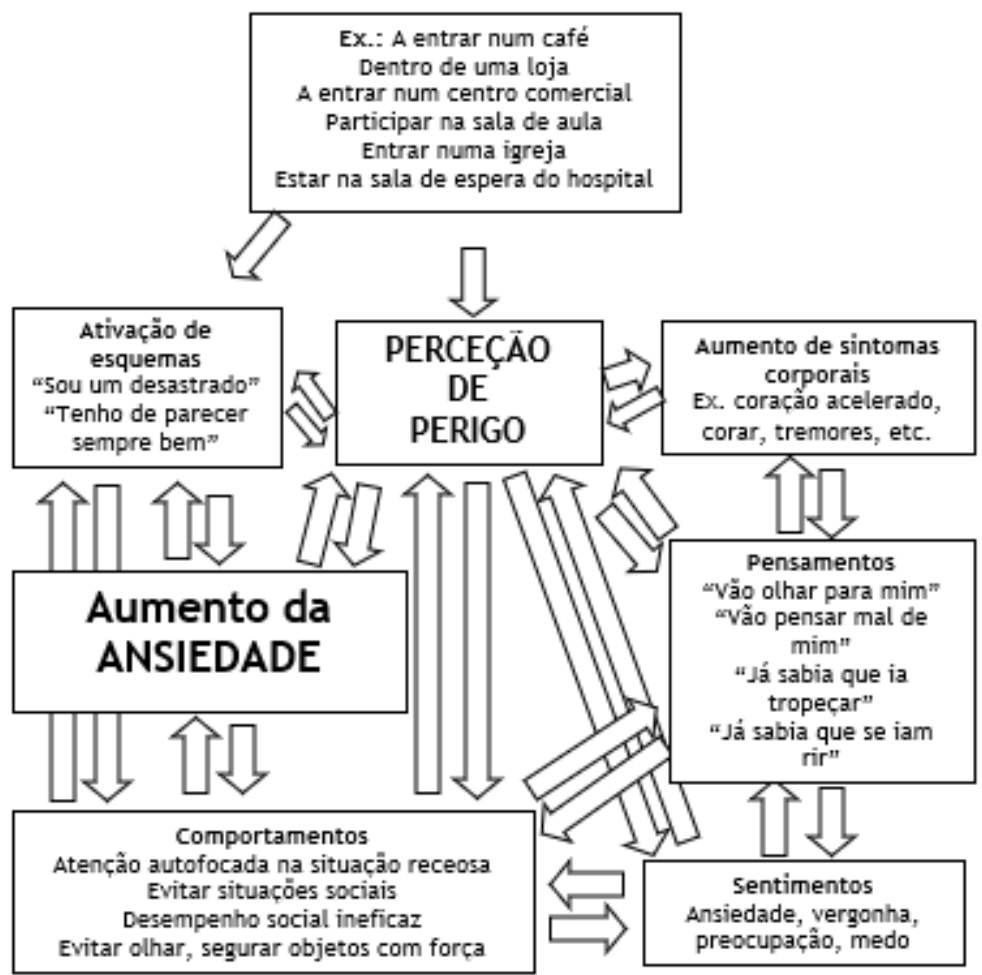

Numa segunda instância, procurou-se estimular a identificação dos PAN relacionados com a probabilidade e severidade de ameaças a nível social. Nesse âmbito, foi pedido a $\mathrm{H}$ um registo das situaçóes, do nível de ansiedade, dos pensamentos, dos comportamentos e da percepção acerca da probabilidade e da severidade dessas situaçôes. Posteriormente, foi feita a análise de evidência confirmatória e desconfirmatória das ameaças sociais, a análise de custo/ benefício, a descatastrofização e a classificação dos erros cognitivos subjacentes. Após essa fase, promoveu-se a geração de interpretações alternativas mais realistas e racionais para as situações, incluindo a reavaliação da probabilidade e da severidade das ameaças com base na análise da evidência efetuada (Clark, \& Beck, 2010).

Numa terceira instância, foi solicitado a $\mathrm{H}$. que começasse a eliminar seus comportamentos de segurança, procurando explicar de que forma cada um deles contribui para a manutenção da ansiedade social (Clark, \& Beck, 2010). Por exemplo, evitar o contato visual faz com que não seja permitido desconfirmar os pensamentos negativos sobre o eventual perigo de olhar nos olhos das pessoas e sobre a forma como estas o interpretam. 
Numa quarta instância, procedeu-se ao uso de estratégias comportamentais, como exposiçóes in vivo, com o objetivo de desconfirmar os pensamentos negativos e os esquemas que contribuem para a manutenção da fobia social. Para isso, e com base na classificação das situações sociais e na sua hierarquização por parte de H., a exposição foi iniciada pelas situações menos geradoras de ansiedade e menos evitadas. No fim, foi analisada a discrepância entre as predições negativas e os resultados reais, no sentido de auxiliar na modificação dos pensamentos e das crenças negativas, bem como no evitamento do recurso futuro à ansiedade antecipatória e na resolução da autópsia (Clark, \& Beck, 2010).

Numa quinta instância, foram trabalhados os esquemas disfuncionais que estão na base do surgimento dos PAN por meio da terapia focada nos esquemas de Young (Young, 1990), dado que se verificaram os EMP de vulnerabilidade ao mal e à doença, fracasso e procura de aprovação. Foram utilizadas técnicas experienciais, tais como recordação de experiências passadas e atuais (ex.: críticas do pai) e expressão emocional mediante diálogos imaginários (ex.: com as pessoas que considera que o vão criticar). Para as crenças apresentadas por H. nesse contexto (ex.: "sou um desastrado"), foi também feita a análise de evidência destas, a identificação e classificação dos erros cognitivos subjacentes, a avaliação das vantagens e desvantagens das respostas de coping (ex.: desvantagens do esforço de satisfazer os outros), o teste de prediçôes e a listagem de afirmaçôes alternativas e mais produtivas (ex.: "só porque tropeço às vezes, não significa que sou desastrado") (Clark, \& Beck, 2010; DeRubeis et al., 2010; Gonçalves, 2004; Martin \& Young, 2010).

Numa sexta e última instância, foi acionado um treino de competências sociais, promovendo-se a assertividade e fornecendo-se estratégias para $\mathrm{H}$. enfrentar, de modo mais eficaz, as situações de índole social (Beidel et al., 2014; Pinto-Gouveia, 2000).

\section{CONCLUSÃO}

Foram efetuadas, ao todo, 61 consultas, sendo que 32 sessões foram destinadas à intervenção psicoterapêutica ao nível da depressão e 29 sessões à intervenção ao nível da ansiedade social. Após a intervenção na depressão, verificaram-se progressos, como melhoria do humor, maior capacidade de questionamento dos PAN, maior recurso a pensamentos racionais e positivos, maior envolvimento em atividades prazerosas, melhoria da qualidade de sono, estabilização do apetite e aumento da capacidade da resolução de problemas. Foi evidente a aprendizagem que $\mathrm{H}$. fez das técnicas cognitivas e comportamentais, com o objetivo de reduzir a sintomatologia depressiva, mas também de servirem de ferramentas a utilizar 
no futuro para lidar com situações problemáticas semelhantes.

A intervenção na fobia social permitiu registar evoluções, tais como redução ligeira da ansiedade, um melhor enfrentamento de situações sociais, a adoção de uma atitude mais assertiva, uma menor utilização de comportamentos reforçadores da fobia social, uma maior capacidade de analisar PAN e uma maior competência para gerar respostas alternativas. A intervenção ao nível da ansiedade social é essencial, pois pode prevenir a ocorrência de perturbações do humor posteriores, tendo em conta a elevada comorbilidade entre a fobia social e a depressão (Kessler, Stang, Wittchen, Stein, \& Walters, 1999).

Quanto aos esquemas centrais de H., foi difícil modificá-los, mas se verificou o esforço por parte do jovem em questionar e perceber a relação que existe entre as crenças e sua forma de pensar, sentir e agir. No período de follow-up (4 sessões quinzenais e 6 mensais), foi importante o trabalho de manutenção do humor, redução do nível de ansiedade, modificação de pensamentos negativos e orientação para o futuro no sentido das estratégias a adotar ante eventuais problemas. Devido à dificuldade característica de $\mathrm{H}$. em gerir a ansiedade, muito devido à postura autoritária do pai e às discussões familiares, foram introduzidas outras técnicas, como a respiração diafragmática e a visualização positiva, e $\mathrm{H}$. foi aconselhado a praticar exercício físico, aumentar sua rede de apoio social e envolver-se em técnicas de meditação (Cabete, Cavaleiro, \& Pinteus, 2003; Sarafino, \& Smith, 2011).

A nível familiar, foi importante uma intervenção com os pais, sensibilizandose para a importância da influência negativa do pai na manutenção dos esquemas de defeito e de procura de aprovação pelos outros, em relação à sua forma de agir com o filho, aos efeitos adversos da violência familiar e à excessiva importância dada ao estatuto social da família. Quanto à mãe, foi alertada no sentido de que a excessiva proteção que exerce para com $\mathrm{H}$. e a preocupação constante com os perigos que lhe podem acontecer são fatores que contribuem para a manutenção dos seus esquemas de fracasso e de vulnerabilidade ao mal e à doença.

Concluindo, este trabalho é limitativo no sentido de que foi exposta apenas a intervenção num caso clínico específico, pelo que não se poderá generalizar à população em geral nem a outros contextos. Contudo, este trabalho veio mostrar que a terapia cognitivo-comportamental é de fato uma modalidade terapêutica útil e eficaz no tratamento psicológico da depressão e da fobia social, corroborando as evidências da literatura. Para além disso, a realização deste estudo de caso poderá fornecer pistas para a realização de mais relatos de experiência profissional posteriores e, ou, estudos subsequentes sobre o uso dessa tipologia terapêutica e sobre a sua eficácia nas perturbações depressivas e na fobia social. 


\section{REFERÊNCIAS}

American Psychiatric Association. (2014). Manual diagnóstico e estatístico das perturbaçôes mentais: DSM-5. (5a ed.). Porto Alegre: Artmed.

Beck, A. T., Rush, A. J., Shaw, B. F., \& Emery, G. (1979). Cognitive therapy of depression. New York: Guilford.

Beidel, D. C., Alfano, C. A., Kofler, M. J., Rao, P. A., Scharfstein, L., \& Sarver, N. W. (2014). The impact of social skills training for social anxiety disorder: a randomized controlled trial. Journal of Anxiety Disorders, 28, 908-918. Recuperado a partir de http://dx.doi.org/10.1016/j.janxdis.2014.09.016

Cabete, D. G., Cavaleiro, A. M., \& Pinteus, M. T. (2003). Visualização: uma Intervenção possível em Psicologia da Saúde. Análise Psicológica, 2(21), 195-200. Recuperado a partir de http://www.scielo.mec.pt/pdf/aps/v21n2/ v21n2a06.pdf

Clark, D. A., \& Beck, A. T. (2010). Cognitive therapy of anxiety disorders: science and practice. New York: Guilford.

Clark, D. M., \& Wells, A. (1995). A cognitive model of social phobia. In R. G. Heimberg, M. R. Liebowitz, D. A. Hope, \& F. R. Schneier (Eds.), Social phobia: diagnosis, assessment and treatment. (pp. 69-93). New York: Guilford.

Derogatis, L. R. (1994). Symptom checklist-90-R (SCL-90-R): administration, scoring, and procedures manual. ( $3^{\mathrm{a}}$ ed.). Minneapolis: National Computer Systems.

DeRubeis, R. J., Webb, C. A., Tang, T. Z., \& Beck, A. T. (2010). Cognitive therapy. In K. S. Dobson (Ed.), Handbook of cognitive-behavioral therapies $\left(3^{\text {rd }}\right.$ ed.) (pp. 277-316). New York: Guilford.

Gonçalves, O. (2004). Terapias cognitivas: teorias e práticas. (4 $4^{\mathrm{a}}$ ed.). Porto: Afrontamento.

Kessler, R. C., Stang, P., Wittchen, H. U., Stein, M., \& Walters, E. E. (1999). Lifetime co-morbidities between social phobia and mood disorders in the US national comorbidity survey. Psychological Medicine, 29(3), 555567. Recuperado a partir de https://www.cambridge.org/core/journals/ psychological-medicine/article/abs/lifetime-comorbidities-between-socialphobia-and-mood-disorders-in-the-us-national-comorbidity-survey/ A0221168D8E18C6CC07F9BF6F69EA494 
Klein, D. F., \& Wender, P. H. (2005). Understanding depression: a complete guide to its diagnosis and treatment. New York: Oxford University Press.

Knapp, P., \& Beck, A. T. (2008). Fundamentos, modelos concetuais, aplicaçóes e pesquisa da terapia cognitiva. Revista Brasileira de Psiquiatria, 30(2), S54-64. Recuperado a partir de http://dx.doi.org/10.1590/S151644462008000600002

Knaus, W. J. (2006). The cognitive behavioral workbook for depression. Oakland: New Harbinger.

Kunzler, L. S., \& Araujo, T. C. C. F. (2013). Cognitive therapy: using a specific technique to improve quality of life and health [Terapia cognitiva: aplicações de uma técnica para qualidade de vida e saúde]. Estudos de Psicologia, 30(2), 267-274. Recuperado a partir de http://dx.doi.org/10.1590/S0103166X2013000200013

Martin, R., \& Young, J. (2010). Schema therapy. In K. S. Dobson (Ed.), Handbook of cognitive-behavioral therapies. (3 ${ }^{\text {rd }}$ ed.). (pp. 277-316). New York: Guilford.

Mascella, V. (2013). Depression: causes and treatment [Depressão: causas e tratamento]. Estudos de Psicologia, 30(2), 303-304. Recuperado a partir de http://dx.doi.org/10.1590/S0103-166X2013000200016

McIntyre, T., \& McIntyre, S. (1995). Inventário de depressão de Beck: versão portuguesa. Braga: Universidade do Minho.

Nezu, A. M., Nezu, C. M., \& D’Zurilla, T. J. (2013). Problem-solving therapy: a treatment manual. New York: Springer.

Nihalani, N., Simionescu, M., \& Dunlop, B. W. (2009). Depression: phenomenology, epidemiology, and pathophysiology. In T. L. Schwartz, \& T. J. Petersen (Eds.), Depression: treatment strategies and management. ( $\left.2^{\text {nd }} \mathrm{Ed}.\right)$. (pp. 1-21). London: Informa UK.

Ogden, J. (2010). Health psychology: a textbook. (5 $5^{\text {th }}$ ed.). Maidenhead: Open University Press, McGraw Hill.

Pinto-Gouveia, J. (2000). Ansiedade social: da timidez à fobia social. Coimbra: Quarteto. 
Pinto-Gouveia, J., Cunha, M., \& Salvador, M. C. (2000). Um protocolo para a avaliação clínica da fobia social através de questionários de auto-resposta. In J. Pinto-Gouveia (Ed.), Ansiedade social: da timidez à fobia social. (pp. 237-258). Coimbra: Quarteto.

Pinto-Gouveia, J., \& Salvador, M. C. (2001). The social interaction anxiety scale and the social fobia scale in the portuguese population. In Anais, 31 Congress of the European Association for Behaviour and Cognitive Therapy, Istambul.

Powell, V. B., Abreu, N., Oliveira, I. R., \& Sudak, D. (2008). Terapia cognitivocomportamental da depressão. Revista Brasileira de Psiquiatria, 30(2), 73-80. Recuperado a partir de http://www.scielo.br/pdf/rbp/v30s2/a04v30s2.pdf

Sanderson, W. C., \& McGinn, L. K. (2001). Cognitive-behavioral therapy of depression. In M. M. Weissman (Ed.), Treatment of depression: bridging the 21st century. (pp. 249-279). Washington: American Psychiatric Press.

Sarafino, E. P., \& Smith, T. W. (2011). Health Psychology: biopsychosocial interactions. ( $7^{\text {th }}$ Ed.). New Jersey: John Wiley \& Sons.

Spielberger, C. D. (1983). Manual for the State-Trait Anxiety Inventory (STAI). Palo Alto: Consulting Psychologists.

Wilkinson, G., Moore, B., \& Moore, P. (2003). Tratar a depressão. Lisboa: Climepsi.

Young, J. E. (1990). Cognitive therapy for personality disorders: a schema-focused approach. Sarasota: Professional Resource Exchange. 